\title{
Study of suspension settling: A approach to determine suspension classification and particle interactions
}

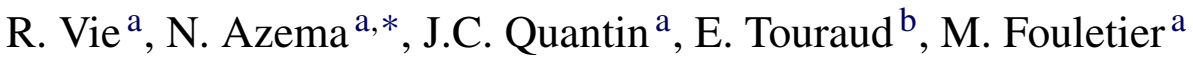

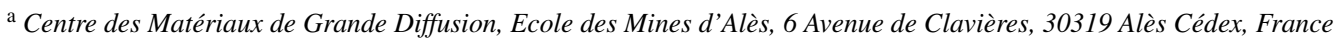 \\ ${ }^{\mathrm{b}}$ Laboratoire du Génie de l'Environnement Industriel, Ecole des Mines d'Alès, 6 Avenue de Clavières, 30319 Alès Cédex, France
}

\begin{abstract}
The properties of mineral suspensions do not evolve linearly with particle mass fraction. For some critical concentrations these properties are abruptly modified due to a change of relative interparticle interaction force prevalence. Using kaolin and glass beads as model materials, physicochemical stability and rheological measurement have been carried out in order to propose a global and discriminant parameter enabling the nature of particle interactions to be identified. Different types of suspensions were studied namely: diluted suspensions, concentrated suspensions and solid suspensions. Physico-chemical stability, studied by optical dispersion analysis (Turbiscan MA 2000) enables the clarification rate to be measured and to propose a new global parameter, the "phase separation index". In addition, rheological analysis was realized by the means of viscous flow measurement. Behaviour of solid suspensions is satisfactorily described by Oswald de Waele power law in the whole range of concentration studied which tends to demonstrate that the interactions remains the same in this range.
\end{abstract}

Keywords: Particle interactions; Classification; Suspension; Kaolin; Settling; Characterisation

\section{Introduction}

Pastes and suspensions are widely used in industrial area, recycling and waste processing. Stability and mechanical properties which mainly depend on particle size, particle shape, interparticle forces and solid concentration, are of practical as well as fundamental interest. The properties of these media do not evolve linearly with solid concentration. A few scientists such as Coussot and Ancey [1] and Tradros [2] distinguished three types of suspensions: diluted suspensions, concentrated suspensions and solid suspensions. This typology is mainly based on the correlation between the prevalent interparticle interactions and the corresponding rheological properties. In the case of supra-colloidal particles (from 1 to $100 \mu \mathrm{m}$ ) which are large enough to neglect Brownian diffusion, each type of suspension has a characteristic settling behaviour governed by specific types of interactions. In a diluted suspension, particles are far from each other and have no colloidal interactions. They settle indi-

\footnotetext{
* Corresponding author. Tel.: +334667853 56; fax: +3346678 5365 .

E-mail address: nathalie.azema@ema.fr (N. Azema).
}

vidually [3]. In a concentrated suspension, particles settle as a more or less consolidated mass with a sharp boundary between settling suspension and supernatant. The suspension behaviour corresponds to a regime known as hindered settling (also called zone settling or mass settling $[3,4]$ ) and water moves in spaces between particles. On the other hand, in solid suspensions, the particles are in contact and settle under compression. Under the influence of gravity the contact structure moves to a more compact one [4,5].

This work proposes a new approach to determine particle interactions through the study of the settling kinetics using an optical analysis. Suspensions containing a high solid mass fraction that cannot be optically analysed were studied by rheological measurements.

\section{Materials and methods}

\subsection{Kaolin suspensions}

Experiments were carried out on kaolin from Prolabo Company (CAS number 1332-58-7) dispersed in demineralised and filtered water in order to control chemical entities in the medium. 


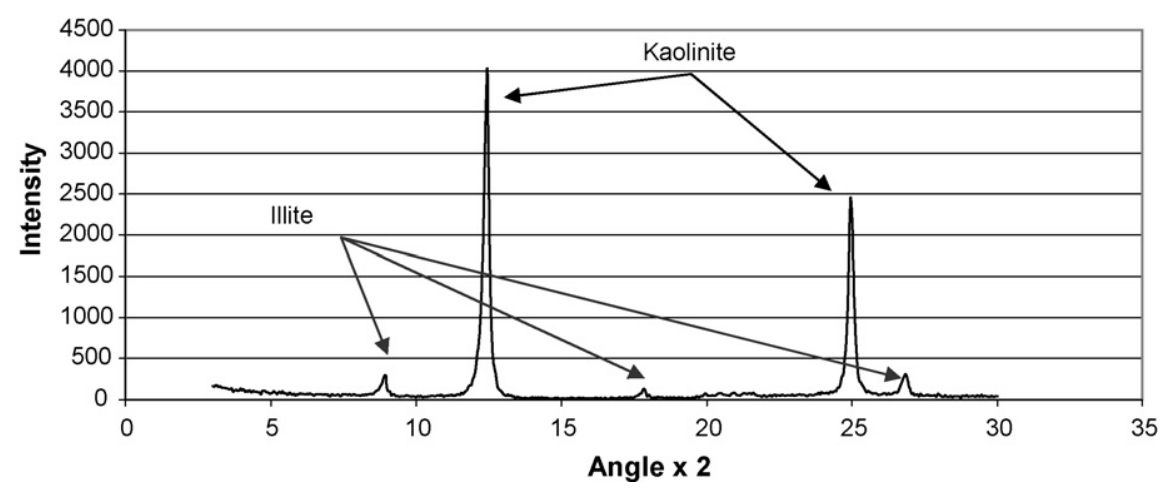

Fig. 1. X-ray diffractogram of kaolin.

Samples were prepared from kaolin dry powder to which distilled water was added until a total weight of $30 \mathrm{~g}$ was reached. The resulting suspensions undergo a $15 \mathrm{~min}$ stiring at a constant speed in order to obtain a homogenous and dispersed medium.

\subsubsection{X-ray analysis of kaolin powder}

In order to characterize the mineralogical composition of the powder, X-ray diffraction analysis was carried out using a Philips PW 1700 instrument. A copper cathode was used. The evaluation program used was: DIFFRACT-AT programmed by M. Socabim.

The X-ray diffractogram represented in Fig. 1 indicates the presence of another kind of clay than kaolinite $\left(\mathrm{Al}_{2} \mathrm{Si}_{2} \mathrm{O}_{5}(\mathrm{OH})_{4}\right)$, the illite. The approximate formula of which is $\left(\mathrm{K}_{0.88} \mathrm{Al}_{2}\left(\mathrm{Si}_{3.12} \mathrm{Al}_{0.88}\right) \mathrm{O}_{10}(\mathrm{OH})_{2}\right.$ according to Rosenberg [6].

The ratio illite/kaolinite was determined using the method proposed by Larray based on X-rays diffraction [7]. This ratio was estimated to be of the order of $2 \pm 1 \%$.

\subsubsection{Size distribution analysis}

Particle size distribution of the kaolin was studied using a LS 230 laser granulometer (Beckman-Coulter). Granulometric distribution shows three modes at $0.3,5.5$ and $13 \mu \mathrm{m}$ as represented in Fig. 2 and a granular spreading $\left(d_{95}-d_{5}\right)$ of $22.6 \mu \mathrm{m}$.

\subsection{Glass microbeads}

In order to get an example of non-cohesive particle suspensions, suspensions based on glass microbeads of various sizes from Potters Europe Society were studied.

\subsection{Multiple light scattering analysis}

The variation of suspension stability as a function of mass fraction was studied at room temperature $\left(21^{\circ} \mathrm{C}\right)$ using the dispersion optical analyser Turbiscan MA 2000 (formulaction) [8].

The sample was introduced in a glass cylindrical cell and analysed using a light beam emitted in near infrared $(850 \mathrm{~nm}$ wavelength) which scans the sedimentation column. The time elapsed between two consecutive scans is chosen as a function of the sedimentation kinetics of the suspension. For example, in the case of concentrated suspensions, the scans were recorded each minute. In the case of diluted suspensions, the scans were recorded each $15 \mathrm{~min}$. Each scans lasts $20 \mathrm{~s}$. Transmitted and backscattered photons are analysed using detectors placed respectively at $0^{\circ}$ and $135^{\circ}$ from the incident beam direction.

\subsection{Strain-controlled rheometry}

Rheological properties at room temperature $\left(21^{\circ} \mathrm{C}\right)$ were determined using a rotational ARES rheometer from TA Instru-

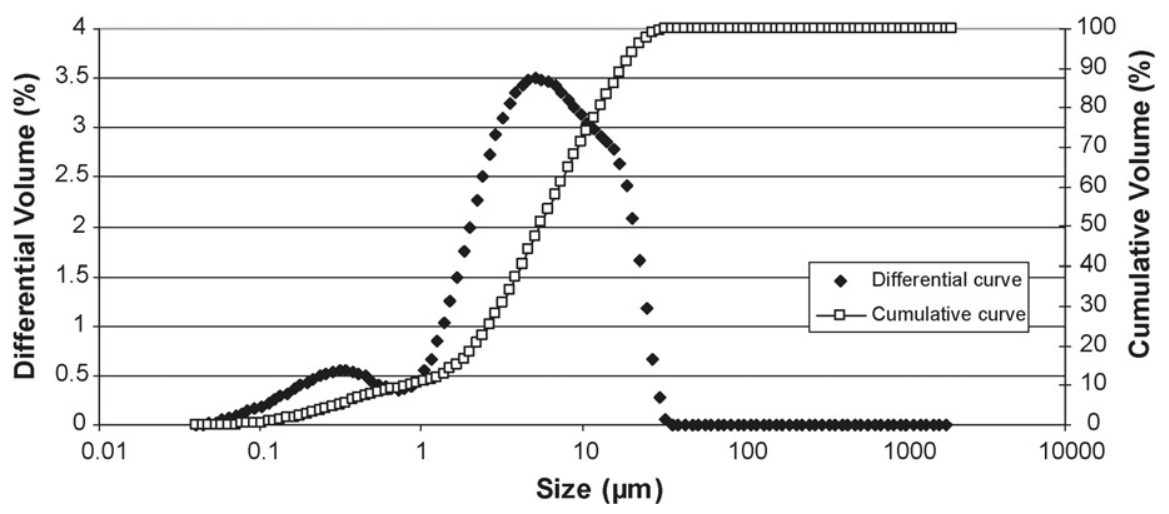

Fig. 2. Particle size distribution of kaolin. 
ments Company. Parallel geometry was employed. According to Coussot and Ancey [1] and Papo et al. recommendations [9], all the measurements were carried out using $50 \mathrm{~mm}$ diameter aluminium serrated plates in order to prevent suspension slipping on the wall.

All the suspensions were initially manually sheared at a high rate while $1 \mathrm{~min}$ in order to suppress the previous rheological history of the sample tested.

\section{Results and discussion}

\subsection{Characterisation of kaolin suspensions: definition of a new global parameter}

\subsubsection{Classification of kaolin suspensions}

Spectra represented in Figs. 3 and 4 correspond respectively to diluted and concentrated suspensions (the sedimentation profile is schematically depicted in the lower part of each figure). The spectrum of a solid suspension is similar to that of a concentrated one. $X$-axis represents height of the tube and $Y$-axis the transmitted or backscattered light percentage. The values in the column on the right part give the correspondence between the recorded trace and the time elapsed. From these spectra it is possible to obtain classical determinations such as clarification rate. To do so, evolution of the clarification signal thickness has been followed as a function of time. Clarification signal thickness was measured at $2 \%$ of transmitted light (Figs. 3 and 4). Fig. 5 represents evolution of the clarification signal thickness as a function of time for two types of suspensions (diluted and concentrated). Clarification rate was obtained from the slope of the curves. Points between 0 and 3 min were ignored because clarification front is not well formed at these times. When the settling velocity was not constant during the measurement (especially in the case of high sedimentation rate) it was evaluated from the linear part of the curve observed in the beginning of the measurement when particle movements are not disturbed by the increase of the concentration.

Nevertheless a new parameter named "phase separation index" (PSI) was proposed in a global characterisation approach. This parameter is defined from the sedimentation study. It is defined (Eq. (1)) as the ratio between the sedimentation column height ( $\mathrm{Hc}$ ) and the sediment height at given sedimentation time, i.e. $15 \mathrm{~min}$ (Hs) multiplicated by the average value of the percentage of backscattered light $(\mathrm{Bs} \%)$ in the zone defined as the sediment (i.e. in Hs):

$\mathrm{PSI}=\left(\frac{\mathrm{Hs}}{\mathrm{Hc}}\right) \times(\mathrm{Bs} \%)$

PSI is a dimensionless parameter. Hc and Hs were determined as indicated in Figs. 3 and 4. PSI takes into account the quantity of matter which is present in sediment because Bs\%, the average backscattering percentage of the sediment, is directly linked to particle volume fraction $(\phi)$ and the particle size $(d)$ [8] as shown in Eqs. (2) and (3):

$\mathrm{Bs} \sim \frac{1}{\left(\lambda^{*}\right)^{1 / 2}}$

$\lambda^{*}(d, \Phi)=\frac{2 d}{\left[3 \phi(1-g) Q_{\mathrm{s}}\right]}$

where $\lambda^{*}$ is the photon transport length in the considered medium (m), $Q_{\mathrm{s}}$ the scattering efficiency factor (dimensionless), and $g$ is the asymmetry factor (dimensionless) [8].

The evolutions of the PSI parameter and of the clarification rate as functions of mass ratio in suspensions are represented in Fig. 6.

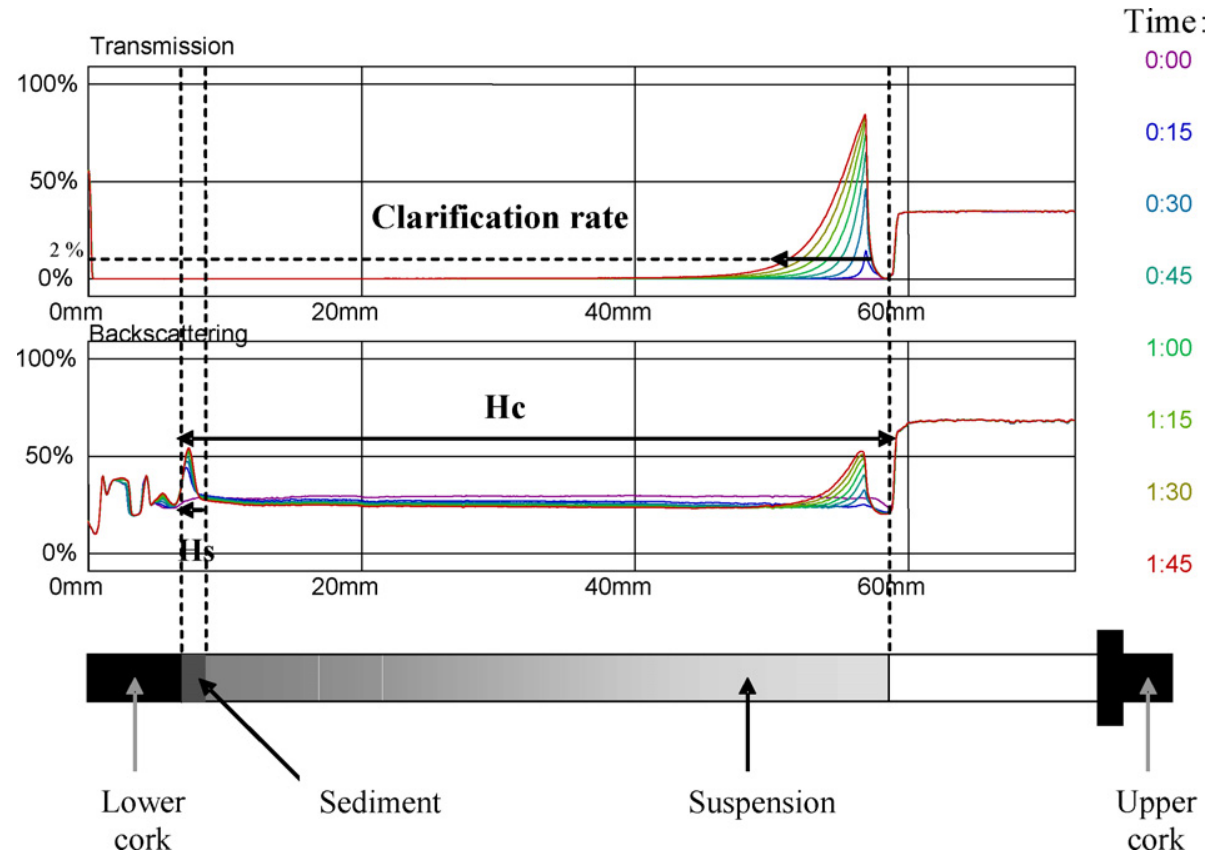

Fig. 3. Example of Turbiscan MA 2000 spectrum of diluted suspensions. 


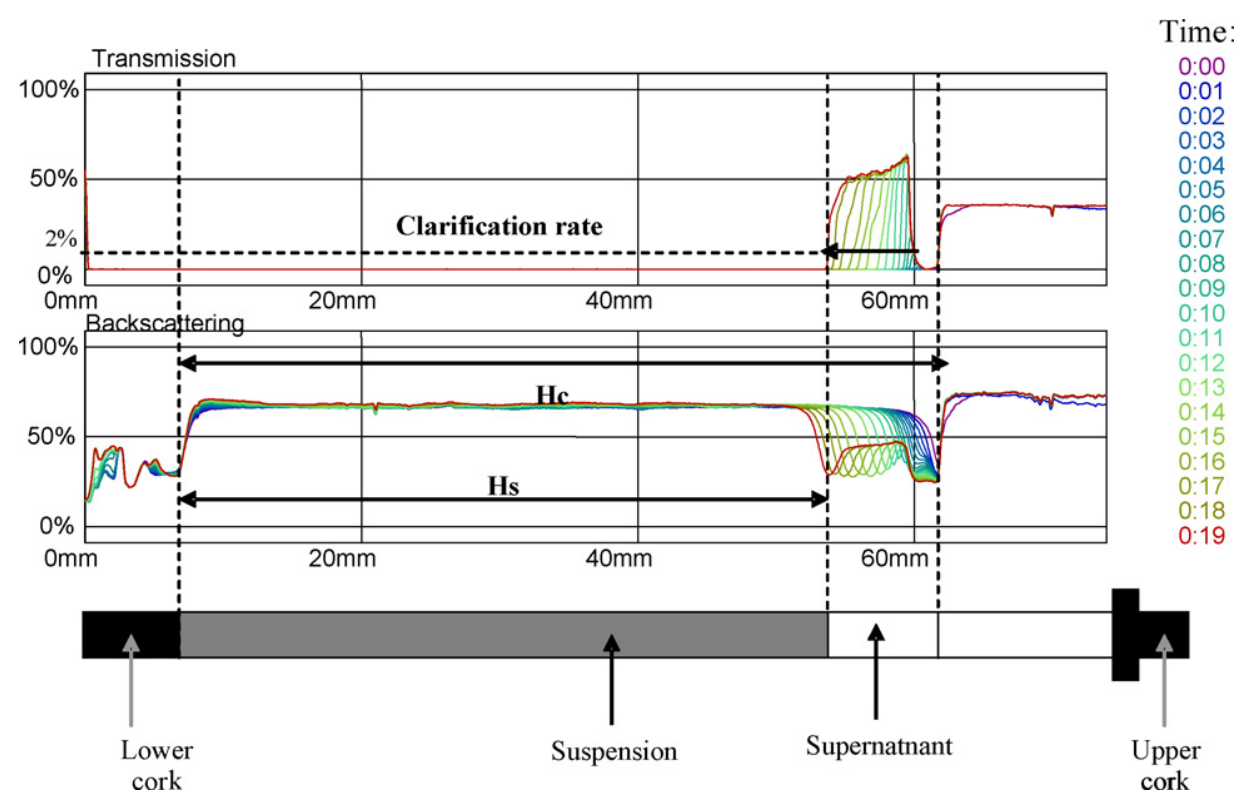

Fig. 4. Example of Turbiscan MA 2000 spectrum of concentrated and solid suspensions.

Clarification rate is generally slow (less than $0.5 \mathrm{~mm} / \mathrm{min}$ ) but undergoes an important increase when the solid fraction is comprised between 4 and $9 \%$. The clarification rate may reach values as high as $5.2 \mathrm{~mm} / \mathrm{min}$. Phase separation index exhibits linear evolution with two discontinuities characterised by a change of slope. Discontinuities do not occur abruptly, so PSI makes possible three zones $\mathrm{A}, \mathrm{B}$, and $\mathrm{C}$ to be distinguished corresponding to the different types of suspensions, i.e. diluted, concentrated and solid suspensions and two intermediate zones.

\subsubsection{Zone A: diluted suspensions (from 0.26 to $1.04 \%$ in} mass fraction). In this part, clarification rate is small and is almost independent of the kaolin concentration. So, in this mass fraction domain, particles do not interact through colloidal forces. This behaviour is typical of the diluted suspensions [1,2]. While settling processes, a transpararency gradient is observed in the column which corresponds to a concentration gradient. But even in the bottom of the tube increase in concentration is not high enough to enable colloidal interactions to occur.
3.1.1.2. Intermediate zone 1 (from 1.8 to $3.5 \%$ in mass fraction). In this domain, clarification rate remains small (less than $0.5 \mathrm{~mm} / \mathrm{min}$ ) and almost independent of the particle concentration but phase separation index increases rapidly. The concentration gradient above-mentioned implies that changes in interparticular force prevalence may occur at these concentrations. Particles are sufficiently close to each other to make possible colloidal interactions to take place. Some agglomerates are formed, then, kinetics of sedimentation in the lower part of the tube is enhanced. The sediment height will be higher too because loose agglomerates occupy a greater volume than its constitutive particles due to their fractal structures [10].

\subsubsection{Zone B: concentrated suspensions (from 4 to $9 \%$ in} mass fraction). Zone $\mathrm{B}$ is characterized by an abrupt increase in the clarification rate. Kaolin particles immediately form agglomerates due to physico-chemical interactions. Forming large structures, the agglomerated particles got a higher sedimentation rate [3]. Moreover, the slope of the PSI variation versus

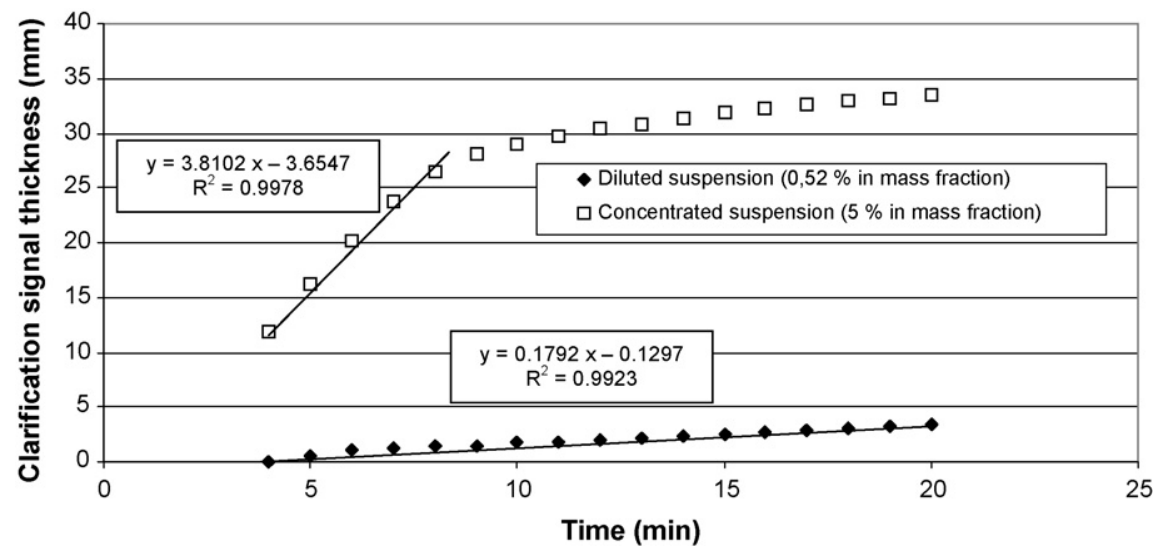

Fig. 5. Determination of clarification rate for a diluted and a concenterated suspension $(0.18$ and $3.8 \mathrm{~mm} / \mathrm{min}$, respectively). 


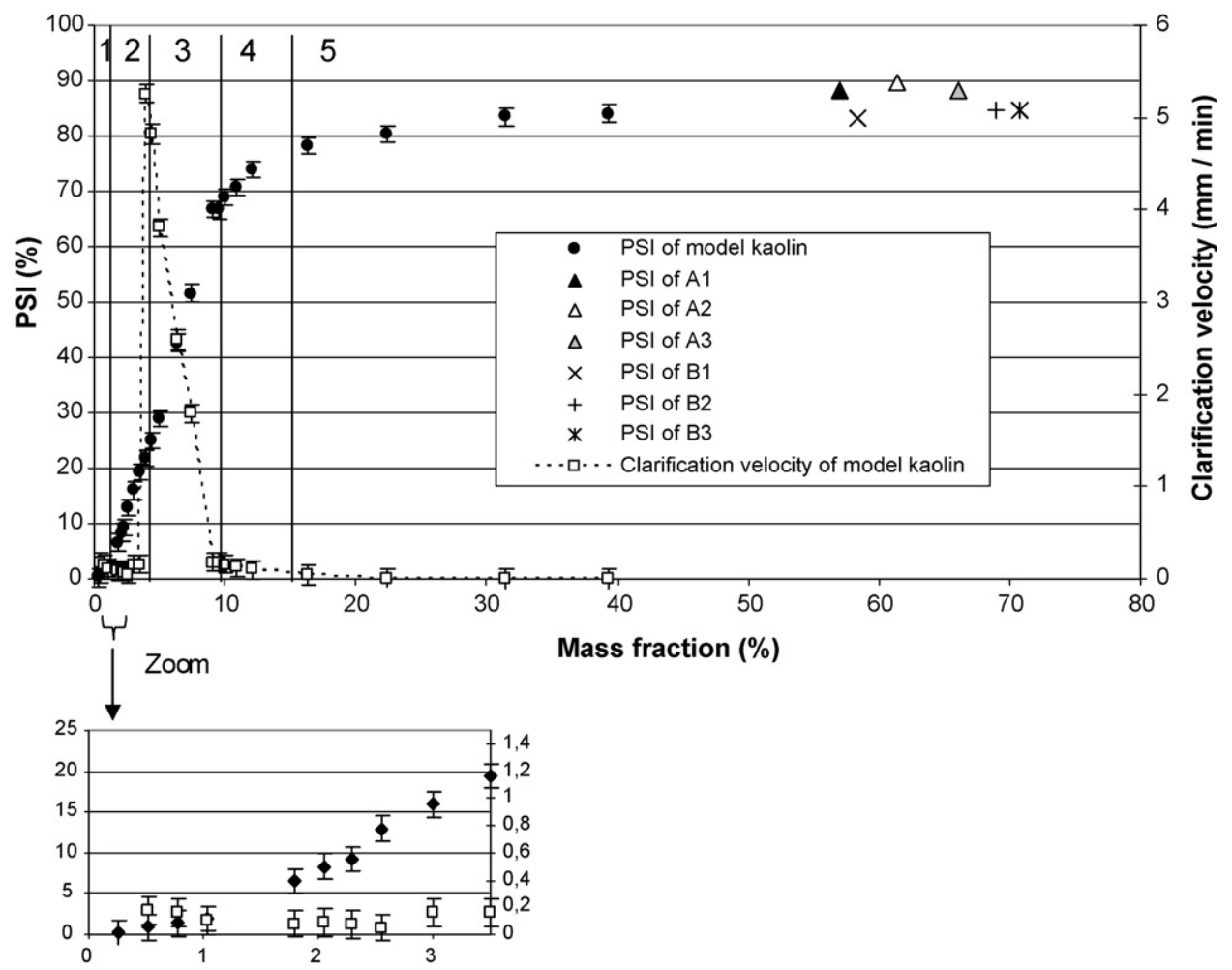

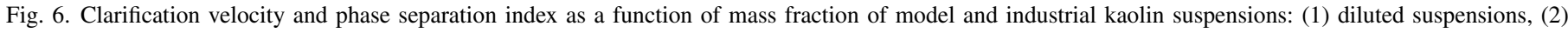
intermediate state 1, (3) concentrated suspensions, (4) intermediate state 2, and (5) solid suspensions.

concentration curve is steeper because the sedimentation mechanism changes radically and "hindered settling" regime occurs. This regime is characterized by a sharp boundary between the suspension and the supernatant [11]. The solids subside as a more or less consolidated structure due to the fact that concentration is large enough to develop agglomerate contact as they settle [12]. Water movement is hindered by solid phase then clarification velocity decreases as a function of mass fraction [13].

\subsubsection{Intermediate zone 2 (from 9.5 to $16 \%$ in mass fraction).} In this zone, clarification velocity is strongly slowed down and PSI evolution is disturbed. The contacts which occur between the agglomerates while sedimentation takes place become more numerous. In fact, by increasing the concentration of solids, agglomerates cannot grow indefinitely without interpenetration $[14,15]$. The agglomerates are then smaller and able to form a more compact structure corresponding to a smaller sediment height which explains that PSI evolution is slowed down.

\subsubsection{Zone C: solid suspensions (from 16 to $40 \%$ in mass frac-} tion). In this domain, all the particles (or agglomerates) form a contact network in the suspension and settling phenomenon can occur only by compression. Sedimentation rates tend to zero, the weight of the particles in the sample is too small to create a compression force high enough to generate a significant ramming in the suspension which is already highly concentrated. Evolution in the PSI is then due to the increase in the backscattering percentage value generated by the higher initial concentration.

\subsubsection{Kaolin suspensions from ceramics industry}

In Fig. 6 the evolution of the phase separation index of industrial samples which belong to ceramics industry is also represented. Samples $\mathrm{A}_{(1,2,3)}$ and $\mathrm{B}_{(1,2,3)}$ are suspensions made from two different kaolins formulated with different additives. The different numbers correspond to differences in formulation and additive concentrations. The lower viscosity of industrial samples is due to their formulation; the addition of dispersing agents made possible measurements to be realised at higher mass fraction $(55-70 \%)$. The PSI values seem to be mainly influenced by the type of kaolin. This parameter makes possible the suspensions made from the two different kaolins A and $\mathrm{B}$ to be differentiated. Clarification velocities corresponding to these samples tend to zero and were not represented in Fig. 6.

Unlike sedimentation velocity, PSI possesses significant and discriminant values in the whole range of mass fractions suspension studied. In the case of model suspensions and the PSI plateau value makes possible to distinguish highly concentrated industrial suspensions made from two different kaolins. Furthermore, this parameter exhibits intermediate behaviour between each type of suspensions. This observation suggests that the transition from a type of settling to another one is not abrupt, but takes place over a narrow range of concentration as asserted by B. Fitch in the case of clarification/hindered settling regime transition [11]. This difference is due to the fact that clarification rate is measured in the top of the tube while PSI is determined in the bottom where concentration can increase while sedimentation takes place. 


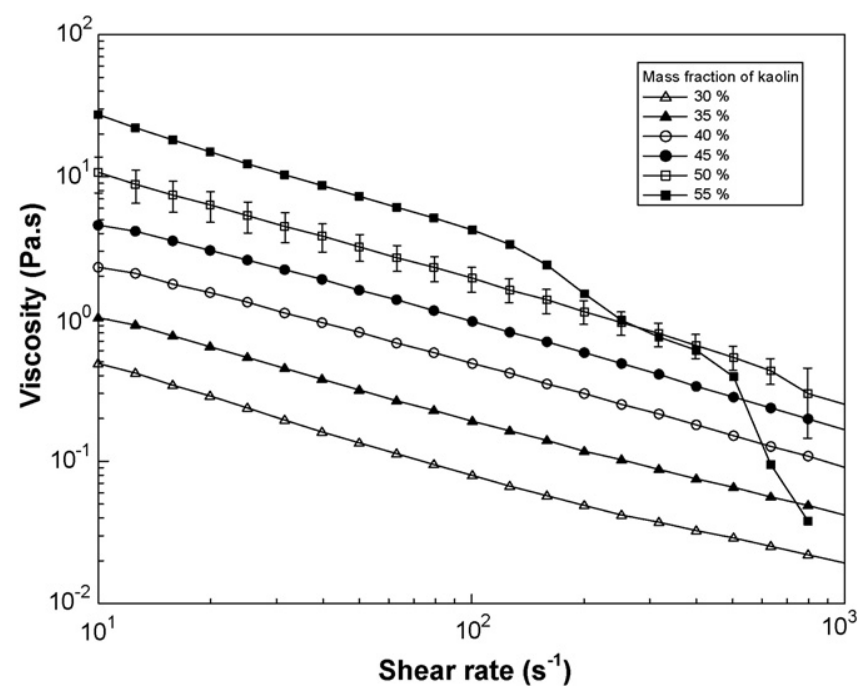

Fig. 7. Flow measurements of kaolin suspensions.

So PSI appears to be more effective than clarification rate in the description of the destabilisation process of suspensions.

\subsection{Study of solid suspensions using viscous flow measurements}

In order to study solid suspensions with a mass fraction higher than $40 \%$ a study of rheological properties of kaolin suspensions as a function of mass fraction was carried out through viscous flow measurements (Fig. 7).

Mass fractions between 30 and 55\% were studied. In this range of concentrations suspension viscosities are too low to be correctly analysed by the detector. Beyond this range, suspensions undergo fracturing phenomenon which makes measurement impossible. As it can be seen in Fig. 7 this disturbing effect occurs at $50 \%$ of mass fraction at the last point and 55\% but only beyond $100 \mathrm{~s}^{-1}$. The major part of these two rheograms is still correct; then, the analysis of these samples remains possible.

All the studied suspensions are shear-thinning, the evolution of their viscosity as a function of shear rate obeys a power law that can be fitted with the Oswald de Weale model using the following equation (Eq. (4)):

$\eta=K(\dot{\gamma})^{n-1}$

where $\eta$ is the viscosity (Pas), $K$ the consistency index ( $\mathrm{Pa} \mathrm{s}^{n}$ ), $\dot{\gamma}$ the shear rate $\left(\mathrm{s}^{-1}\right)$, and $n$ is the flow index $(0<n<1$ for shear-thinning fluids; dimensionless).

This relation between shear rate and viscosity is typically linked to progressive destruction of fractal structured agglomerates $[10,16]$ induced by shearing $[17,18]$.

Consistency index $(K)$ evolves exponentially with mass fraction (Fig. 8). Flow index ( $n$ ) which indicates the departure from Newtonian behaviour does not exhibit a significant evolution, its value is $0.28 \pm 0.05$ for all the samples, so the straight line like rheograms (on a bi-logarithmic scale) in Fig. 7 can be considered as having the same slope.

No discontinuities have been noticed in the evolution of these two parameters in the Oswald de Waele power law with mass fraction changes. These results lead to the conclusion that there is no change in the suspension type in this range of concentration.

\subsection{Observation of the agglomeration state by microscopic observations}

Existence of different discontinuities in the evolution of PSI suggests that particle organisation is different in each type of suspensions. In order to observe these structural differences, three suspensions have been deposited on an aluminium surface, air dried and observed using a high resolution environmental electronic microscope Quanta 200 FEG from FEI Company. Each of them belongs to one of the three types of suspensions. Their mass fractions are respectively $1 \%$ for the diluted one, $6.3 \%$ for the concentrated one and $22.5 \%$ for the solid one. As it can be seen in Fig. 9 the state of agglomeration after drying is different in each type of suspension.

Edges of the particles from the diluted suspensions appear clearly and it is possible to observe the stacking of plate-like shape of kaolin particles as it can be done with samples of dry powder before adding water. This is an indication that the agglomeration phenomenon is almost non-existent in this type

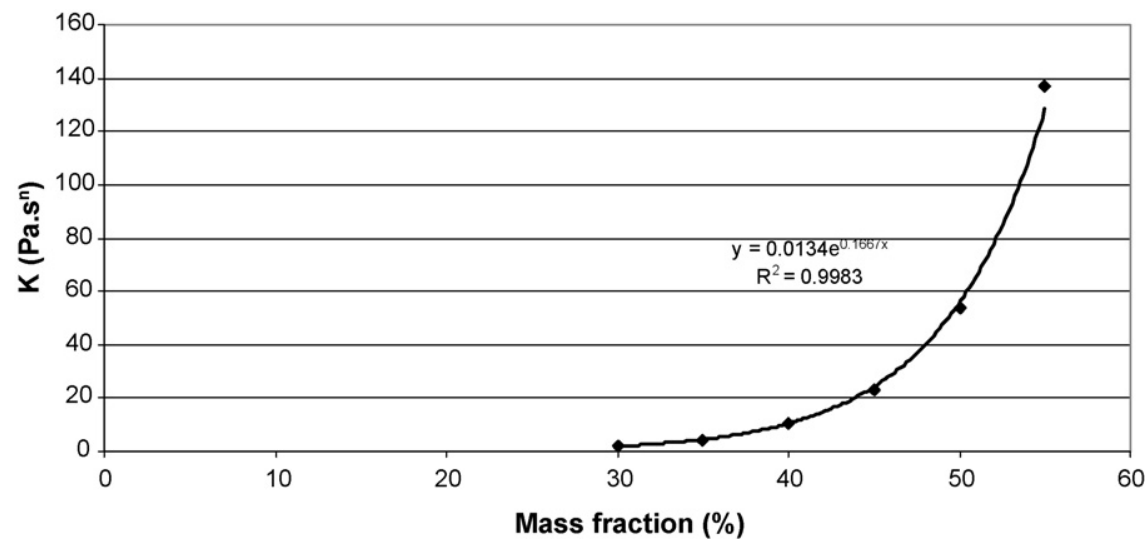

Fig. 8. Coefficient of consistency as a function of mass fraction of kaolin suspensions. 

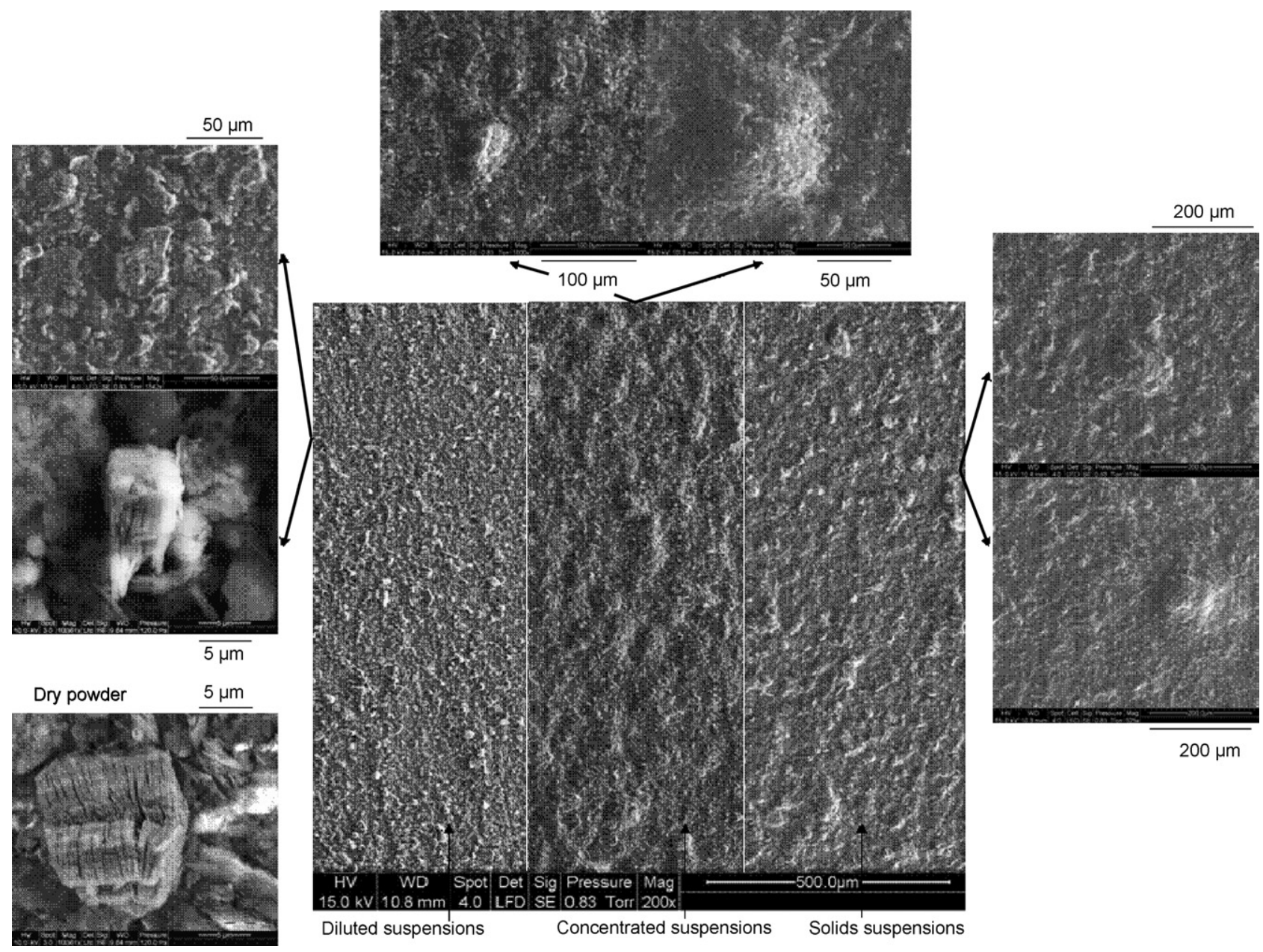

Fig. 9. MEB micrographs of the different types of kaolin suspensions.

of suspensions. In the case of concentrated suspensions, it is possible to observe agglomerates having various sizes (between 40 and $90 \mu \mathrm{m})$. It can be inferred that all particles while in suspension have been interacting with their neighbours. Small particles are agglomerated with bigger ones, which explains why edges of the particles are more difficult to identify. The structure of the solid suspension is comparable with that of concentrated suspensions, but agglomerates are smaller (between 15 and $60 \mu \mathrm{m}$ ). While agglomerates are formed in the suspension, they enter in contact, due to high concentration and hinder their own growing $[13,14]$.

\subsection{Influence of particle interactions on PSI variations}

In order to identify the influence of the cohesive particle interaction strength on the evolution of PSI, non-cohesive glass beads suspensions and kaolin suspensions at different $\mathrm{pH}$ have been studied.

\subsubsection{Influence of the $\mathrm{pH}$ on kaolin suspensions}

As it has been previously asserted the settling process of the suspensions depends on the interparticle interactions. The strength of these different interactions can be deeply modified with a $\mathrm{pH}$ variation of the dispersing fluid. Generally, acid water supports the agglomeration of kaolin particles, whereas basic water acts like a deflocculant [19].

This tendency is confirmed in the media which were studied in this work as indicated by the evolution of the electrophoresis mobility as a function of the $\mathrm{pH}$ represented in Fig. 10. These measurements have been carried out using a DELSA 440 zetameter from Coulter Company.

At low pH surface charge of the particles is small; repulsion forces are not efficient, then, agglomeration phenomenon is strong. At higher $\mathrm{pH}$ surface charge is stronger and agglomeration in the suspension is less important. The evolution of the phase separation index as a function of mass fraction was studied at $\mathrm{pH} 4$ and 11 and compared with results obtained at neutral pH (Fig. 11).

The acid suspensions exhibit a strong increase of phase separation index even for the lower values of mass fractions. The agglomeration phenomenon is strong and seems to be important even in the lowest particle concentration range which has been studied. Diluted suspensions domain has disappeared. Agglomeration occurs in the same time as sedimentation phenomenon takes place even in domain of the lowest concentration studied. In fact, the suspensions with a mass fraction of 0.52 and $1.04 \%$ are in intermediate state 1 . Beyond these concentrations up to $8 \%$ media is in the concentrated suspensions 


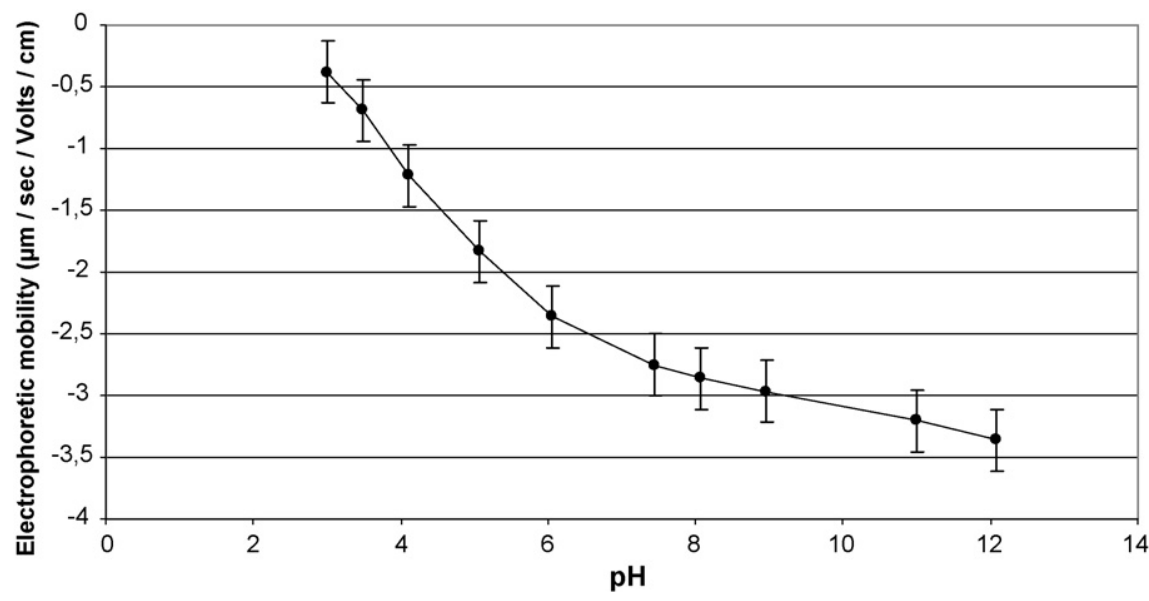

Fig. 10. Electrophoresis mobility variations as a function of the $\mathrm{pH}$ of kaolin suspensions.

domain. Solid suspension domain, at $\mathrm{pH} 4$, is reached at slightly lower values than for neutral $\mathrm{pH}$ because of stronger agglomeration phenomenon. Agglomerates formed are bigger $[15,16]$ and begin to enter in contact at lower particle concentration.

The low values of the phase separation index for kaolin concentration of $0.3-12 \%$ at $\mathrm{pH} 11$ show that attraction between particles is very weak. Any increase of the slope of PSI which would indicate concentrated suspension domain can by observed. This shows that at alkaline $\mathrm{pH}$, agglomeration phenomenon is limited. Intermediate zone 2 seems to have disappeared too. In fact, beyond $15 \%$ in mass fraction, particles are sufficiently close to each other to form loose agglomerates which in turn form contact network $[15,16]$. Agglomeration and network formation occurs nearly at the same time because, in alkaline media, agglomeration is possible at high mass fractions. Then, diluted suspensions become solids suspensions when mass fraction increases in a very narrow range of concentration.

\subsubsection{Example of PSI evolution of non-cohesive particle suspensions}

PSI evolutions of glass beads suspensions of different size were studied as a function of mass fraction as it is represented in Fig. 11. In the range of sphere radius studied, PSI values change with particle size but its evolution remains the same. Without any agglomeration phenomenon PSI evolution is deeply modified. It increases linearly from 0.4 to $48 \%$ and does not exhibit any discontinuity. Concentrated suspension domain and intermediate state 1 are not observed.

Beyond $75 \%$ samples were comparable to wet sand with a very high viscosity and it was impossible to implement the experiments. Unlike the kaolin suspensions, contact network in glass bead suspensions is composed of rigid particles and not loose agglomerates. This explains why solid suspension domain is reached at so high concentration with glass beads suspensions and why viscosity is so strongly increased.

Between 50 and $70 \%$ in mass fraction PSI behaviour is not really linear especially for suspensions made from $0-50 \mu \mathrm{m}$

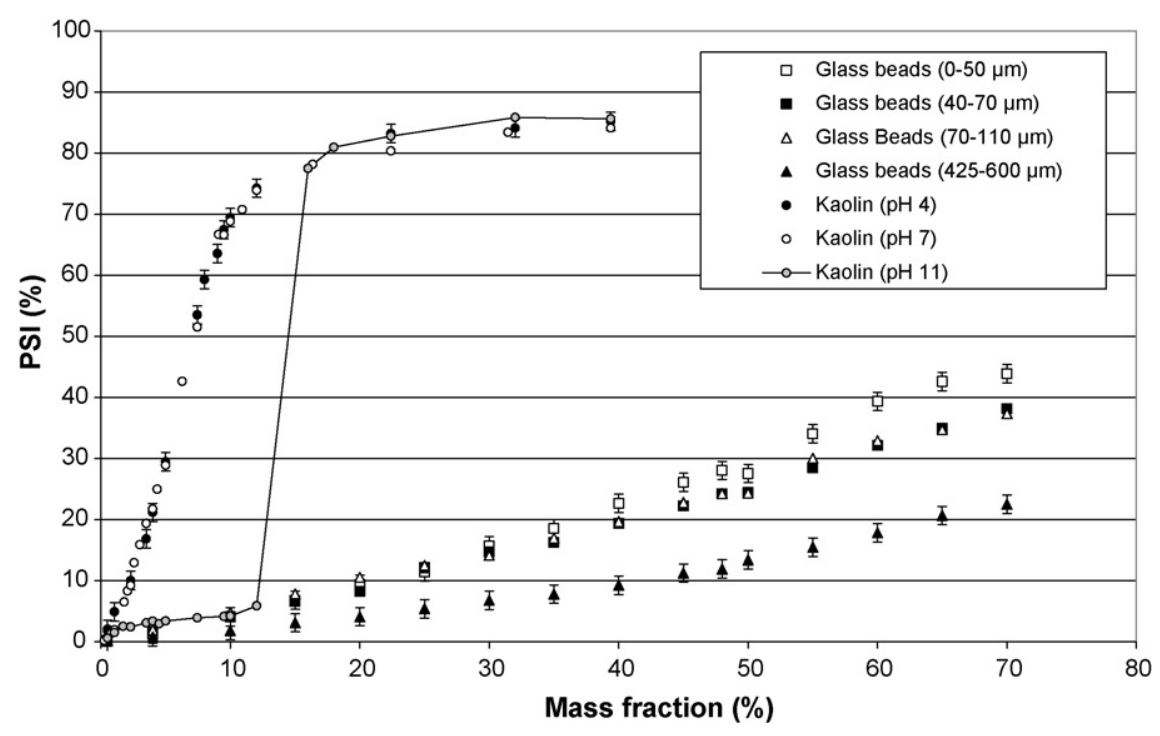

Fig. 11. Phase separation index of the different studied suspensions as a function of mass fraction. 
glass beads. This is due to the backscattering signal which arrives at saturation at these high concentrations.

\section{Conclusion}

For some critical concentrations properties of suspensions are abruptly modified due to a change in their interparticle interactions as it have been shown with clarification rate. Destabilisation properties are not modified so sharply because concentration evolves while settling processes.

Phase separation index which depends on average size of particles and solid concentration satisfactorily describes the evolution of these destabilisation phenomena as a function of mass fraction. Starting from variations of the PSI, the three types of suspensions described in scientific press and two intermediate zones could be distinguished. It make also possible to give discriminant values for all the types of suspensions from 0.1 to $40 \%$ in mass fraction for model samples and up to $70 \%$ to industrial samples which contain surfactants.

Through results obtained for non-cohesive glass beads, kaolin and the influence of the $\mathrm{pH}$ it has been shown that PSI is sensitive to particles granulometry, chemical nature of the solid phase, liquid phase composition and especially particles interactions. It can be proposed, then, to study efficiency of the formulation of a suspension or evaluate agglomeration tendency of a medium.

PSI results are supplemented by rheological tests which made possible higher mass fractions in model media to be studied. Suspensions of kaolin can be characterized by viscous flow measurements in the concentration range from 30 to $55 \%$. $K$ parameter from Oswald de Weale can be related to mass fraction in the medium. Its evolution does not indicate any change in particle organisation after the transition to the solid suspension domain identified from stability study. Thus, Turbiscan MA 2000 is suitable to indentification and characterisation of stability of suspensions. These results make possible a classification of these complex fluids which is principally based on the nature of particles interactions.

\section{References}

[1] P. Coussot, C. Ancey, Rhéophysique des pâtes et des suspensions, EDP Sciences, Les Ulis, 1999.

[2] Th.F. Tradros, Correlation of viscoelastic properties of stable and flocculated suspensions with their interparticle interactions, Adv. Colloid Interf. Sci. 68 (1996) 97-200.

[3] B. Fitch, Sedimentation of flocculent suspensions: state of the art, AIChE J. 25 (6) (1979) 913-930.

[4] D.A. Dahlstrom, B. Fitch, Thickening, in: N.L. Weiss (Ed.), SME Processing Handbook, AIME, New York, 1985, pp. 9-2-9-14.

[5] R.G. Holdich, G. Butt, Compression and channelling in gravity sedimenting systems, Miner. Eng. 9 (1) (1996) 115-131.

[6] P.E. Rosenberg, Am. Miner. 87 (2002) 103-107.

[7] J. Larray, Recherche d'une méthode de dosage quantitative des kaolinites et des illites par diffraction X, thesis, Université Pierre et Marie Curie-Paris VI and Ecole Nationale Supérieure des Mines de Paris, 1981.

[8] O. Mengual, G. Meunier, I. Cayre, K. Peuch, P. Snabre, TURBISCAN MA 2000: multiple light scattering measurement for concentrated emulsion and suspension instability analysis, Talanta 50 (2) (1999) 445-456

[9] A. Papo, L. Piani, R. Ricceri, Sodium tripolyposphate and polyphosphate as dispersing agents for kaolin suspensions: rheological characterization, Colloids Surf. A 201 (2002) 219-230.

[10] J. Gregory, The density of particle aggregates, Water Sci. Technol. 36 (4) (1997) 1-13.

[11] B. Fitch, Sedimentation process fundamentals, Trans. Am. Inst. Miner. Eng. 223 (1962) 129-137.

[12] J.R. Karl, S.A. Wells, Numerical model of sedimentation/thickening with inertial effects, J. Environ. Sci. Eng. 125 (9) (1999) 792-805.

[13] R. Font, M.L. Laveda, Desgn method of continuous thickener from semibath tests of sedimentation, Chem. Eng. Sci. (1996) 5007-5015.

[14] P. Sandkühler, J. Sefcik, M. Morbidelli, Kinetics of gel formation in dilute dispersions with strong attractive particle interactions, Adv. Colloid Interf. Sci. 108-109 (2004) 133-143.

[15] P. Snabre, P. Mills, I. Rheology of weakly flocculated suspensions of rigid particle, J. Phys. III France 6 (1996) 1811-1834.

[16] X. Jia, D.J. Wedlock, R.A. Williams, Simulation of simultaneous aggregation and sedimentation, Miner. Eng. 13 (2000) 1349-1360.

[17] Q. Huang, W. Li, M. Gu, P. Chen, H. Zhang, Y. Jin, Effect of blend ratio on rheological properties of aqueous $\mathrm{SiC}$ suspensions, J. Eur. Ceram. Soc. 24 (2004) 2157-2161.

[18] L. Bergström, Shear thinning and shear thickening of concentrated ceramic suspensions, Colloids Surf. A 133 (1998) 151-155.

[19] O. Cuisset, Propriétés électrocinétiques des particules argileuses. Application de la méthode électrophorétique aux problèmes d'environnement et d'identification des sols, Laboratoire Central des Ponts et Chaussées, 1980. 